\title{
A COMPARISON OF THE CENTIMETRE-TO-SUBMILLIMETRE CONTINUUM SPECTRA OF BL LACERTAE OBJECTS AND FLAT SPECTRUM RADIO QUASARS
}

\author{
J.A. STEVENS and S.J. LITCHFIELD \\ Centre for Astrophysics, University of Central Lancashire, Preston PR1 2HE, UK \\ E.I. ROBSON \\ Joint Astronomy Centre, 660 N. A'ohōkū Place, University Park, Hilo, Hawaii 96720, USA \\ W. K. GEAR \\ Royal Observatory, Blackford Hill, Edinburgh EHg $3 H J, U K$ \\ and \\ D.H. HUGHES \\ Department of Physics, Nuclear Physics Laboratory, Keble Road, Oxford OX1 3RH, UK
}

\begin{abstract}
A comparison of the centimetre to submillimetre continuum spectra of 22 BL Lacertae objects and 24 flat-spectrum radio quasars (FSRQ) has been conducted in order to search for systematic differences between the two classes. The same overall spectral shape is found for all sources and it is concluded that the same basic physical model applies to the continuum emission over this frequency range in both cases. There is clear evidence, however, for the BL Lacs to have flatter high frequency spectra and this difference is reconciled with an intrinsic difference in the underlying jets of the two classes.
\end{abstract}

The quasi-simultaneous multifrequency continuum spectra (see Gear et al. 1993) of each object was analysed by fitting two-point spectral indices to the 5-37 GHz points and the $37-270 \mathrm{GHz}$ points. In addition, a weighted fit was made to the $375,270,230$ and $150 \mathrm{GHz}$ data which describes the optically thin spectral index. A 1-D Kolmogorov-Smirnov (KS) test showed no difference in the $5-37 \mathrm{GHz}$ twopoint spectral indices of BL Lacs and FSRQs. However, significant differences were found between the 37-270 GHz two-point spectral index populations (98.8\%) and between the optically thin spectral index populations (99.5\%), with the BL Lacs having the flatter slopes.

Multi-epoch data, which were available for many sources, showed that the derived spectral indices were highly variable. The optically thin spectral indices were thus separated into two categories; those describing the sources in a high state and those describing the sources in a low state. Re-applying the K-S test, a separation in the populations was still found when the sources are in the low state, but this disappears when the sources are flaring. It is concluded that the observed difference in spectral indices between BL Lacs and FSRQs is due to an intrinsic difference in the underlying jets.

\section{References}

Gear, W. K. et al. 1993, Monthly Notices of the RAS, in press

T. J.-L. Courvoisier and A. Blecha: Multi-Wavelength Continuum Emission of AGN, 523.

(C) 1994 IAU. Printed in the Netherlands. 